\title{
Effects of Photoactivation in Bleaching with Hydrogen Peroxide. Spectrophotometric Evaluation
}

\author{
Lo Giudice $\mathrm{G}^{1}$, Cicciù $\mathrm{D}^{2}$, Cervino $\mathrm{G}^{2}$, Lizio $\mathrm{A}^{3}$, Panarello $\mathrm{C}^{4}$ and Cicciù $\mathbf{M}^{5 *}$ \\ ${ }^{1}$ Professor of Conservative Dentistry, Dental School, Messina University, Messina \\ ${ }^{2}$ Head and Director, Department of Odontostomatology, Dental School, Messina University, Messina \\ ${ }^{3}$ Resident Department of Odontostomatology, Dental School, Messina University, Messina \\ ${ }^{4}$ Resident Department of Odontostomatology, Dental School, Messina University, Messina \\ ${ }^{5}$ Assistant Professor of Oral Surgery, Dental School, Messina University, Messina
}

\begin{abstract}
Objective: Purpose of this study is to point out the effectiveness of a bleaching technique consisting of hydrogen peroxide $38 \%$ applied with and without photo-activation with a LED lamp.
\end{abstract}

Study design: Nine groups, each consisting of two adjacent teeth extracted from the same patient for periodontal disease, have been involved in the study. Similar features in color characterized all teeth considered. The first tooth of each couple was treated by the use of the LED lamp (KromaOgna WS25 ${ }^{\mathrm{TM}}$ ) and the second one without photoactivation. The bleaching agent consist of a gel made of hydrogen peroxide $38 \%$, oxalic acid and ortho-phosphoric acid and a gel activator composed of strontium chloride and calcium hydroxide.

Results: The analysis of colorimetric values underlines how bleached teeth treated with photo activation showed a $67 \%$ of positive changes in color while the remaining $33 \%$ did not go through any variation.

Conclusion: The data of the study clearly evidence that the use of the LED lamp for the whitening procedure increases the effectiveness of the bleaching material if compared to the technique with no photo-activation.

\section{Keywords: Bleaching; Whitening Agent; LED lamp}

\section{Introduction}

The term bleaching means the chemical destruction of the chromophore compounds present in organic and inorganic groups through a redox reaction. Bleaching agent can decolorize a double bonds substratum, aromatics and quinolone systems. In dentistry, teeth whitening procedures include the use of chemicals such as hydrogen peroxide and carbamide peroxide [1,2]. Regarding the chemical reaction, it consists in the saturation or the substitution of double bonds of the chromophore groups, present at the level of organic and inorganic compounds of dental tissue, with single bonds, through the release of nascent oxygen, resulting from the reaction of decomposition of bleaching agents [2,3]. The transformation of the pigments in low molecular weight compounds, result in a lighter and brighter tooth color for the different reflection of the incident light [3]. The whitening treatment is a medical procedure that is not free of complications including the possibility that the peroxide can cause changes in tooth structure, as shown by various in vitro research $[4,5]$ For example hydrogen peroxide $38 \%$, has a strong oxidizing effect that could damage the enamel. The carbamide peroxide 35\% (hydrogen peroxide $11.4 \%$ plus urea), because of the action of urea that is divided in carbondioxide and ammonia, could cause changes in interprismatic enamel areas.

The urea could denature the enamel protein structure determining structural and morphological changes through the degradation of organic molecules such as amelogenins. Another negative effect of urea is linked to its alkalinity that while raising the $\mathrm{pH}$ of bleaching agents reduces their demineralizing properties.

Moreover the bleaching action due to structural changes, gives way to an increase in dentin sensitivity. This symptom generally resolves after two or three days, but could be prolonged and assume the form of a real dental disease that shows with pain, generally localized in one or more teeth, evoked by stimuli of various nature (thermal, chemical or osmotic). The sensitivity, linked to the passage of peroxide through enamel and dentin, arises in few minutes, which takes the form of a reversible pulpitis caused by the flow of dentinal fluid, changing osmolarity without apparent damage to the pulp [6]. Discomfort has been recorded rarely. A possible irritation associated with gingival edema and up to rare cases of ulcerations by direct contact of the gel with the mucosa, which should, if properly applied, be avoided by using dam $[5,6]$. There all indications to the whitening treatment are represented by discoloration or pigmentation that are not necessarily linked to the quality of oral hygiene. Actually, throughout the time, teeth change color because of morpho structural dental changes caused

\begin{tabular}{|c|c|}
\hline \multicolumn{2}{|c|}{ Endogenous discolorations } \\
\hline Pre-eruptive & Post-eruptive \\
\hline Imperfect dentinogenesis & Decalcifications \\
\hline Imperfect amelogenesis & Carious lesions \\
\hline Dentinal dysplasia & Enamel erosion \\
\hline Hyperbilirubinemia & Filling materials \\
\hline Phenylketonuria & Dental traumas \\
\hline Haematic diseases & Endodontic treatments \\
\hline Endocrine diseases & \\
\hline Dental fluorosis & \\
\hline Tetracycline discolorations & \\
\hline
\end{tabular}

Table 1: Classification of intrinsic discoloration

*Corresponding author: Marco Cicciù, Department of Oral Surgery,Denta School, Messina University, Via Consolare Valeria 98100, Messina, Italy, Tel: 0039 090221 6920; E-mail: acromarco@yahoo.it

Received November 10, 2011; Accepted November 21, 2011; Published November 22, 2011

Citation: Lo Giudice G, Cicciù D, Cervino G, Lizio A, Panarello C, et al. (2011) Effects of Photoactivation in Bleaching with Hydrogen Peroxide.Spectrophotometric Evaluation. Dentistry 1:111. doi:10.4172/2161-1122.1000111

Copyright: (c) 2011 Lo Giudice G, et al. This is an open-access article distributed under the terms of the Creative Commons Attribution License, which permits unrestricted use, distribution, and reproduction in any medium, provided the original author and source are credited. 
by age. Moreover lifestyle, food and drinks, smoking, pharmacological therapies and dental diseases may determine typical chromatic changes, linked as well to constitutional and genetic factors [7]. The discolorationcan be classified according to etiology into:Endogenous or intrinsic discoloration and exogenous or extrinsic discoloration. The intrinsic discoloration according to the etiologycan be divided into two types: pre-eruptive andpost-eruptive (Table 1).

These can be partially resolved with oral hygiene or having one or moreteeth whitening treatments but sometimes the final solution consists of a dental restoration or prosthesis.

- The exogenous or extrinsic discoloration is easier to resolve because their development occurson teeth surface. However, relapses are frequent. These kinds of discoloration are usuallyassociated with several factors, including:

- Dental plaque

- Tartarus

- Dietary factors

- Habits

- Employment

- Mouth wash with chlorhexidine and stannous fluoride

The techniques used in bleaching treatment can be divided into home bleaching and professional bleaching, and in that context is possible to use various photo activation tools to enhance the efficacy of bleaching. The emission of light can be achieved through the use of halogen, plasma, laser and LED lamps that are the preferred. The aim of this research is to evaluate the effectiveness of a bleaching agent consisting of hydrogen peroxide $38 \%$ used with and without photoactivation with a LED lamp.

\section{Material and Methods}

Our research was carried out on samples of nine groups, each consisting of two adjacent teeth extracted from the same patient for periodontal disease and with similar features in color. Exclusion criteria are the presence of aesthetic restoration or past treatments of bleaching. The samples were sectioned at the cemento-enamel junction, and subjected to treatment of scaling and polishing and then stored in a dry environment to be later rehydrated at the time of treatment. The bleaching treatment was performed on the buccal surface of each tooth element, on the first tooth of each couple through the use of the LED lamp (KromaOgna WS25 ${ }^{\mathrm{mi}}$ ) and on the second one without photoactivation.

The bleaching agent used, consists in two self-mixing syringes containing a gel made of hydrogen peroxide $38 \%$, oxalic acid and ortho-phosphoric acid and a gel activator composed of strontium chloride and calcium hydroxide.

After placing the teeth on a silicon base in the treatment of

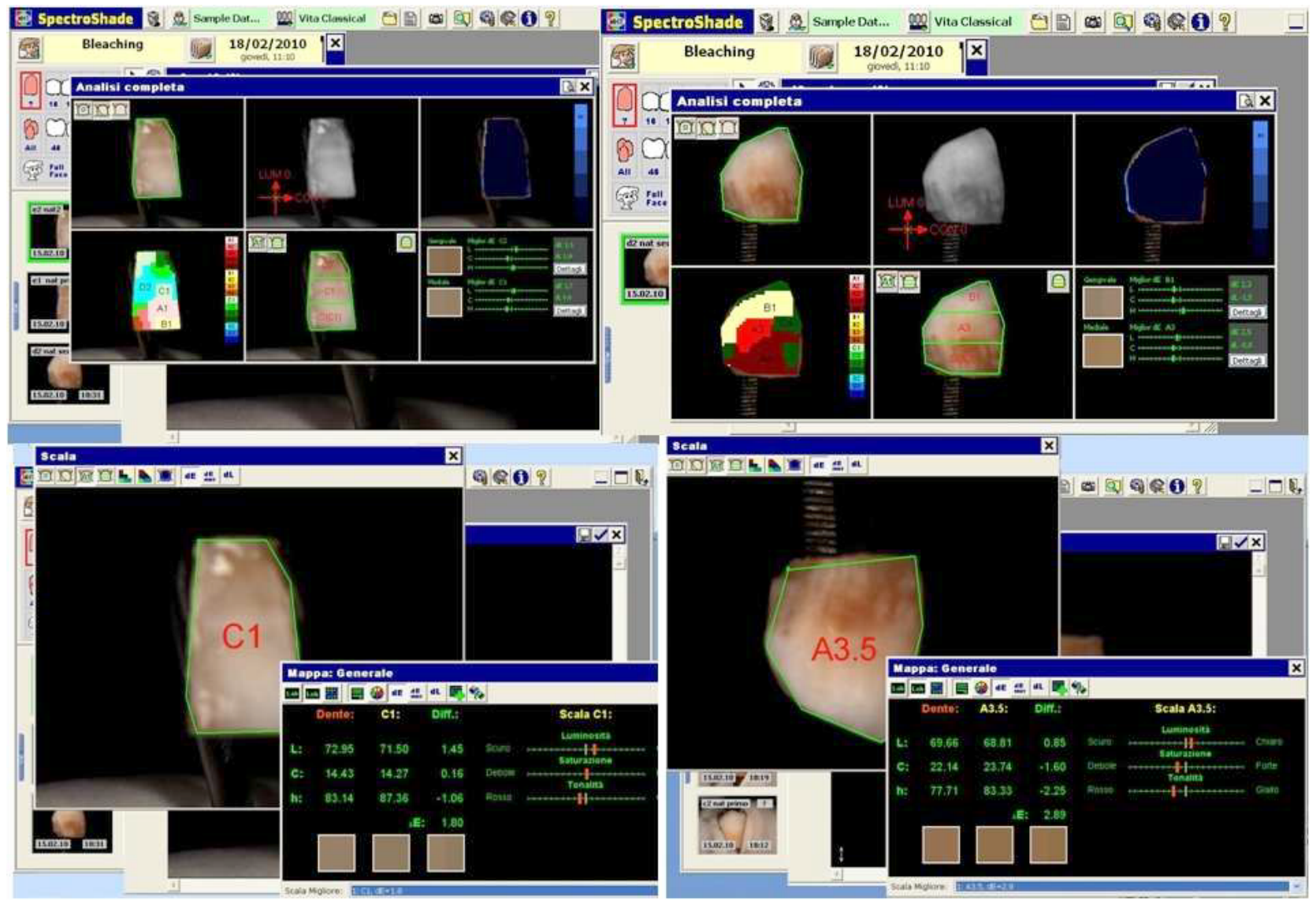

Figure 1: Spectrophotometric evaluation (Spectroshade ${ }^{\mathrm{TM}}$ 
bleaching without photo activation, samples were subjected to three consecutive cycles of bleaching for 15' each, through the application of the gel on the labial surface. The same system was performed during the photo-activated treatment. The lamp used (WS25-Kroma Ogna ${ }^{\mathrm{Tu}}$ ) in the whitening treatment is made up of 14 LEDs ( 2 ultraviolet, 4 infrared and 8 white) with a total power of $60 \mathrm{~W}$, a luminous intensity of $30.000 \mathrm{~mW}$ and a wavelength of $495 \mathrm{~nm}$.

Letters from A to I have been used for group identification. Each teeth whitened with photo activation was included in subgroup L, the remaining in subgroup NL. The chromatic evaluations were performed both before and after the whitening treatment through the use of chromatic scale (Vita Lumin ${ }^{\text {Tux }}$ ) and by using a spectrophotometer (SpectroShade ${ }^{\mathrm{mm}}$ ) for the objective assessment of the value, chroma and hue of the sample (Figure 1).

A photographic control before and after bleaching was performed using the same method; three measurements were made for each item to standardize the readings and to prevent changes in instrument reading. Then the average was calculated by converting the alphanumeric codes of the colors in numbers, according to the conversion table proposed by Brenna [8] (Table 2).

Devices such as reflectance spectrophotometers that typically contain a diffraction grating that splits light into its constituent wavelengths may valuate the amount of light absorbed or reflected by a material at each wavelength in the visible part of the spectrum. The differences of L-lightness (brightness or value), C-chroma (saturation or chroma), $\mathrm{H}$-hue (hue, or tint) were calculated to evaluate the $\Delta \mathrm{E}$. Considering that $\mathrm{L}^{*} \mathrm{a}^{*} \mathrm{~b}^{*}$ values have a perceptual meaning: $\mathrm{L}^{*}$ is the lightness which relates to the physical intensity of a colour, whilst $a^{*}$ and $\mathrm{b}^{*}$ are coordinates on the red-green and yellow-blue colour axes respectively. This scheme is designed such that a constant difference in colour, DE, defined by the Euclidean distance in CIELAB space, thus::

$$
\Delta \mathrm{E}=\sqrt{ } \Delta \mathrm{L}^{*_{2}}+\Delta \mathrm{a}^{* 2}+\Delta \mathrm{b}^{* 2}
$$

This is the objective parameter, which confirms the effectiveness of the treatment.

For $\Delta \mathrm{E}>3$ the differences are significant and are easily distinguished by the untrained eye. For $2<\Delta \mathrm{E}<3$ differences exist but are not well visible. For $\Delta \mathrm{E}<2$, the human eye is unable to make any distinction.

\section{Results}

An analysis of Table 3, Figure 2, which summarize the colorimetric values, shows that bleached teeth with photo activation in the $67 \%$ of the cases had positive changes in color and the remaining $33 \%$ did not go through any variation. As for teeth whitened without the use of the lamp, 56\% had a positive color change and the remaining $44 \%$ do not show any change.

The maximum change obtained by the lamp bleaching is equal to 12

\begin{tabular}{|c|c|c|c|c|c|c|c|c|c|c|c|c|c|c|c|}
\hline B1 & A1 & B2 & D2 & A2 & C1 & C2 & D3 & A3 & C4 & B3 & A3,5 & B4 & C3 & A4 & D4 \\
\hline 16 & 15 & 14 & 13 & 12 & 11 & 10 & 9 & 8 & 7 & 6 & 5 & 4 & 3 & 2 & 1 \\
\hline
\end{tabular}

Table 2: Conversion table (By Brenna F et Al. $)^{7}$

\begin{tabular}{|c|c|c|c|c|c|c|}
\hline \multirow{3}{*}{ Groups } & \multicolumn{4}{|c|}{ Chromatic scale values } & \multicolumn{2}{|c|}{ Spectrophotometer. Values $(\Delta \mathbf{e})$} \\
\hline & \multicolumn{2}{|c|}{$\begin{array}{l}\text { Photoactivated } \\
\text { Bleaching }\end{array}$} & \multicolumn{2}{|c|}{ No photoactivated bleaching } & \multirow[t]{2}{*}{ Photoactivated bleaching } & \multirow[t]{2}{*}{ No photoactivated bleaching } \\
\hline & Pre-bleaching & Post bleaching & Pre-bleaching & Post-bleaching & & \\
\hline A & (A1) 15 & (A1)15 & (B1) 16 & (B1) 16 & 2,28 & 1,75 \\
\hline B & (A1) 15 & (A1)15 & (A1) 15 & (A1) 15 & 9,73 & 3,47 \\
\hline C & (A2) 12 & (B2)14 & (A2) 12 & (A2) 12 & 4,01 & 2,7 \\
\hline D & (A2) 12 & (A1)15 & (B3) 6 & (D2) 13 & 8,06 & 5,37 \\
\hline$E$ & (C3) 3 & (A1)15 & (D2) 13 & (A1) 15 & 11,76 & 5,09 \\
\hline $\mathrm{F}$ & (A3) 8 & (A1)15 & (D2) 13 & (A1) 15 & 7,99 & 6,93 \\
\hline G & (A1) 15 & (A1)15 & (A1) 15 & (A1) 15 & 3,21 & 4,89 \\
\hline $\mathrm{H}$ & (A2) 12 & (A1)15 & (A2) 12 & (A1) 15 & 12,06 & 7,35 \\
\hline 1 & (C1) 11 & (B1)16 & (C4) 7 & (D3) 9 & 10,63 & 7,78 \\
\hline
\end{tabular}

Table 3: Pre and post bleaching values. Chromatic and spectrophotometric evaluation.

\begin{tabular}{|c|c|c|c|c|c|c|}
\hline & \multicolumn{4}{|c|}{ Chromatic scale values } & \multicolumn{2}{|c|}{ Spectrophotometer. Values $(\Delta \mathrm{E})$} \\
\hline & \multicolumn{2}{|c|}{$\begin{array}{l}\text { Photoactivated } \\
\text { Bleaching }\end{array}$} & \multicolumn{2}{|c|}{ No photoactivated bleaching } & \multirow{2}{*}{ Photoactivated bleaching } & \multirow{2}{*}{ No photoactivated bleaching } \\
\hline & Pre-bleaching & Post bleaching & Pre-bleaching & Post-bleaching & & \\
\hline Mean & 11,44 & 15,00 & 12,11 & 13,8 & 7,74 & 5,03 \\
\hline Std. Error & 1,30 & 0,16 & 1,16 & 0,73 & 1,24 & 0,70 \\
\hline Difference & \multicolumn{2}{|c|}{3,56} & \multicolumn{2}{|c|}{1,69} & \multicolumn{2}{|c|}{2,71} \\
\hline$P$ value & \multicolumn{2}{|c|}{0,027} & \multicolumn{2}{|c|}{0,047} & \multicolumn{2}{|c|}{0,01} \\
\hline
\end{tabular}

Table 4: Chromatic and spectrophotometric evaluation. Pre and post bleaching. (mean values, standard error, mean differences and p. Value) 
(from C3 to A1) against the value of 7 obtained without the lamp (from B3 to D2). Paired T Test was used to investigate the differences between pre and post bleaching values with and without photo-activation. The differences were significant $(\mathrm{P}<0.05)$ (Table 4$)$.

The average difference obtained by photo-activated bleaching is equal to 3.56 against 1.69 obtained by the treatment without the lamp. (Table 4).

Analysis of Table 3 - 6 shows that, for the group bleached with the lamp, $88.89 \%$ gives an effective result $(\Delta \mathrm{E}>3)$ and only in $11.11 \%$ the treatment is unsatisfactory $(2<\Delta \mathrm{E}<3)$. For the group bleached without the lamp, in the $77.78 \%$ treatment was effective in $11.11 \%$ it was not very satisfactory $(2<\Delta \mathrm{E}<3)$ and in the remaining cases $(11.11 \%)$ not acceptable $(\Delta \mathrm{E}<2)$. The maximum value of treatment with lamp is equal to 12.06 against 7.78 in the case of treatment without photo-activation.

The average value of $\Delta \mathrm{E}$ for the group photo-activated is equal to 7.74 while for the group bleached without lamp is equal to 5.03 (Table $4)$. The difference $(2.71)$ was significant $(\mathrm{P}<0.05)$ (Table 4$)$.

\section{Discussion}

Our study performed an evaluation about the real bleaching effectiveness of a gel made of $38 \%$ hydrogen peroxide, oxalic acid and ortho-phosphoric acid and an activator gel composed of strontium chloride and calcium hydroxide with and without photoactivation. The use of LED lamp in bleaching is reported in literature, even if there are no several objective studies [8]. To avoid the insertion of excessive variables into the study, the whitening effect determined by two different techniques has been evaluated by measurements pre and post bleaching carried on contiguous teeth extracted in the same patient [8]. The evaluation used tint, chrome and value as referential

WHITENING WITH LAMP

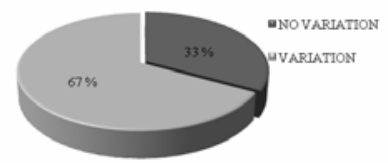

Figure 2: Chart of changes in colour in whitened groups (vitalumin).

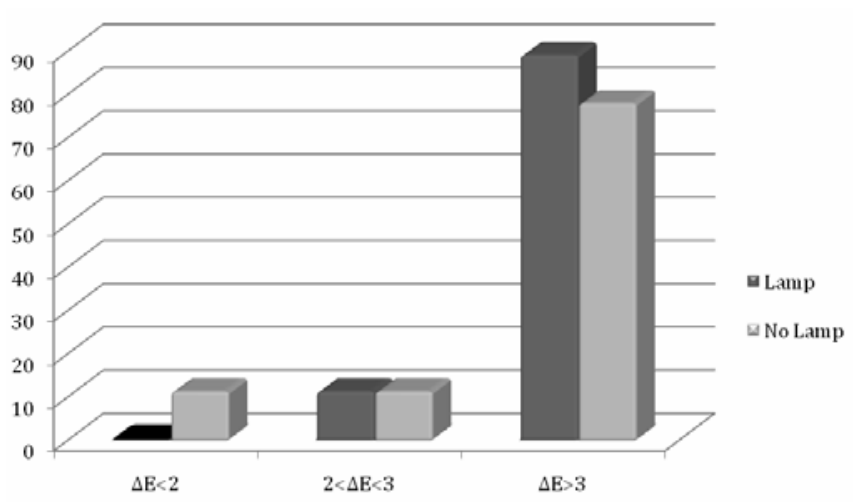

Figure 3: Spectrophotometric evaluation. Valutation (\%) of bleaching effectiveness $(\Delta \mathrm{E}>3,2<\Delta \mathrm{E}<3, \Delta \mathrm{E}<2)$ in the group bleached with or without lamp. parameters to objectify the determination of dental color (Commission Internationale de l'Èclairage) that results from light reflection on an object and allow the quantification of chromatic variation obtained by the treatment.

According to Guan [9] that analyzed the sensitively and accuracy of imaging approach inthe evaluation of bleaching was used two tooth color measurement systems: a visual and a spectrophotometric assessment. These two instrumental approaches have a good correlation although the spectrophotometry valuation underestimates values of the CIE whiteness index and the measurements on the VITA Lumin vacuum shade guide is subject to variations related to the sensitivity operator. [7,10]. Furthermore, the use of spectrophotometer permits the determination of $\Delta \mathrm{E}$, the parameter which confirms the effectiveness of the treatment. The data obtained with the color scales were derived converting the alpha-numeric codes of the colors in numbers for a statistically assessable cross-validation.

The objective evaluations obtained through the spectrophotometric and the colorimetric scale measurements both show the real effectiveness of the bleaching gel tested in our study on discolored teeth. Moreover bleaching was found in the most of teeth in both groups $[67 \%(\mathrm{~L})$ and $56 \%(\mathrm{NL})]$ and the average $\Delta \mathrm{E}$ calculated was $5.03 \pm 0.70(\mathrm{NL})$ and $7.74 \pm 1.24$ (L). The positive color variation of teeth treated with photo activation is evident both in the values noticed from spectrophotometric measurements and with color scale evaluation but this effectiveness is verified in the gingival third of the crown or in the dental elements with an higher chroma $[10,11]$.

Moreover, the data about bleaching demonstrate a different sensibility of teeth to the gel action. The values observed both before and after bleaching in the same tooth change range from 0 to 13 on the chromatic scale and between 1.75 to 7.78 intervals on spectrophotometric values $(\Delta \mathrm{E})$

The comparative evaluation between photoactivated and no photoactivated bleaching, is statistically significant for $\Delta \mathrm{E}$ values while it result not significant for the chromatic scale values. This evidence is probably linked to the sensitivity of the spectrophotometric survey that is able to identify not visible chromatic differences.

Even if the measurements after bleaching in both groups showed remarkable improvements, these colour variations are not always visible to a naked eye. The human eye is not able to detect the difference between a tooth bleached with the photo-activated technique and a tooth bleached with conventional techniques, if the $\Delta \mathrm{E}$ value is lower than 2, and moreover is not able to detect the light difference that can be observed in the various thirds of the crown (incisal, medium and cervical).

Actually there is a greater effectiveness in the gingival third than in the incisal region; this is due to the reduced surface extent, the narrow enamel-dentin thickness and the unfavorable light incidence in the incisal region. Consequently the variations in the cervical level are the most evident for the observer.

Our study shows how the use of the LED lamp for the whitening procedure increases the effectiveness of the bleaching material if compared to the technique without photo-activation [12-15]. However to the difference statistically stressed in the spectrophotometric data doesn't correspond similar clinical evidence. Accordingly to the study data, it seems that the LED lamp application strongly improve the 
Citation: Lo Giudice G, Cicciù D, Cervino G, Lizio A, Panarello C, et al. (2011) Effects of Photoactivation in Bleaching with Hydrogen Peroxide. Spectrophotometric Evaluation. Dentistry 1:111. doi:10.4172/2161-1122.1000111

bleaching procedure.

\section{References}

1. Perdigao J, Baratier LN, Arcari GM (2004) Contemporary trends and techniques in tooth whitening: a review. PractProcedAesthetica Dent 16: 185-192.

2. Zaragoza VMT (1984) Bleaching of vital teeth: technique. EstoModeo9: 7-30.

3. Gerlach R., Zhou X (2001) Vital bleaching with whitening strips: summary of clinical research on effectiveness and tolerability. J Dent Contemporary Practice 15:1-16.

4. Rotstein I (1993) Role of catalase in the elimination of residual hydrogen peroxide following tooth bleaching. J Endod19: 567-569.

5. Lewinstein I, Rotstein I (1992) Effect of trichloracetic acid on the microhardness and surface morphology of human dentin and enamel. Endod Dent Traumatol 8: $16-20$.

6. Flynn J, Galloway R, Orchardson R (1985) The incidence of 'hypersensitive' teeth in the West of Scotland. J Dent $13: 230-236$.

7. Horn DJ, Bulan-Brady J, Hicks ML (1998) Sphere spectrophotometer versus human evaluation of tooth shade. J Endod 24: 786-790.
8. Brenna F, Giani S, Striuli S, Tagliabue A (2007) Sbiancamento professionale: luce-assistito contro le tecniche tradizionali. II DentistaModerno 4: 36-62.

9. Guan YH, Lath DL, Lilley TH, Willmot DR, Marlow I, et al.(2005)The measurement of tooth whiteness by image analysis and spectrophotometry: a comparison. J Oral Rehabil 32: 7-15

10. Joiner A, Hopkinson I, Deng Y, Westland S (2008) A review of tooth colour and whiteness.J Dent 1: s2-7

11. Browing WD, Hackman ST, Pohjola RM (2002) Evaluation of an intra-ora Spectrofotometer. Medical College of Georgia, School of Dentistry, USA IADR/ AADR/CADR $80^{\text {th }}$ General Session

12. Luk K, Tam L, Hubert M (2004) Effect of light energy on peroxide tooth bleaching. J Am Dent Assoc135: 194-201.

13. Walsh LJ (2003) The current status of laser application dentistry. Aust Dent $J$ 48:146-155.

14. Feinman RA, Madray G, Yarborough D (1991) Chemical, optical and physiologic mechanism of bleaching product: a review. Pract Periodontics Aesthet Dent 3 : $32-36$.

15. Pohjola RM, Browning WD, Hackman ST, Myers ML, Downey MC (2002) Sensitivity and tooth whitening agents.J EsthetRestorDent 14: 85-91. 